\title{
NOBLE GASES IN TERRESTRIAL PLANETS: EVIDENCE FOR COMETARY IMPACTS?
}

\author{
TOBIAS OWEN \\ Institute for Astronomy \\ University of Hawaii \\ Honolulu, Hawaii 96822 U.S.A.
}

\author{
AKIVA BAR-NUN and IDIT KLEINFELD \\ Department of Geophysics and Planetary Sciences \\ Tel Aviv University, Tel Aviv 69978, Israel
}

ABSTRACT. The possible role of comets in bringing volatiles to the inner planets is investigated by means of laboratory studies of the ability of ice to trap gases at low temperatures. The pattern of the heavy noble gases formed in the atmosphere of Venus can be explained by the impact of a planetesimal composed of ices formed in the range of 20 to $30 \mathrm{~K}$. The noble gas patterns on Mars and Earth are less explicable by cometary bombardment alone.

\section{Introduction}

The abundances of the non-radiogenic noble gases in the atmospheres of Venus, Earth and Mars, as summarized in Table 1, pose several problems:

(a) On Venus, the ratio ${ }^{84} \mathrm{Kr} / 36 \mathrm{Ar}$ and the upper limit on ${ }^{132} \mathrm{Xe} / 36 \mathrm{Ar}$ resemble the solar abundance pattern, whereas on Earth and Mars, these ratios are much higher.

(b) On Venus, the amount of $36 \mathrm{Ar}$ (per gram of the planet) is 100 times larger than that on Earth, while on Mars, it is 165 times smaller than that on Earth.

(c) Yet the ${ }^{20} \mathrm{Ne} / 36 \mathrm{Ar}$ on Venus is only about twice that on Earth and Mars.

(d) The ${ }^{20} \mathrm{Ne} / 22 \mathrm{Ne}$ on Venus is $11.9 \pm 0.7$, while on Earth, it is 9.8. (The neon isotope ratio was not measured directly in the Martian atmosphere. In the SNC meteorites-widely considered to have a Martian origin${ }^{20} \mathrm{Ne} / 22 \mathrm{Ne}=10.1 \pm 0.7$ (Wiens et al. 1986).)

Any attempt to account for the noble gas patterns on the three planets will have to explain all four problems satisfactorily.

Several authors have attempted to interpret these noble-gas abundance patterns and isotopic ratios in terms of a late-accreting meteoritic VENEER (e.g., Anders and Owen 1977). Carbonaceous chondrites contain noble gases in abundance ratios that resemble those formed on Earth, but not in detail. For example, the xenon on Earth is depleted (or enriched in the meteorite) by roughly a factor of 10 . In any case, the meteoritic pattern cannot account for the abundances found on Venus (Donahue and Pollack 1983). 
Table 1. Ratio of Abundances of ${ }^{36} \mathrm{Ar}$ to Other Noble Gases*

\begin{tabular}{lcccccc}
\hline & & & & \multicolumn{3}{c}{ Enrichment Factors } \\
& ${ }^{20} \mathrm{Ne}$ & ${ }^{36} \mathrm{Ar}$ & ${ }^{84} \mathrm{Kr}$ & ${ }^{132} \mathrm{Xe}$ & ${ }^{84} \mathrm{Kr} /{ }^{36} \mathrm{Ar}$ & ${ }^{132} \mathrm{Xe} /{ }^{36} \mathrm{Ar}$ \\
\hline Sun & 0.027 & 1 & 3,300 & 69,000 & 1 & 1 \\
Venus & $3.3 \pm 1.5$ & 1 & $1,200^{+3000}$ & $>3,000$ & $2.8_{-2.0}^{+0.3}$ & $<23$ \\
Earth & 1.8 & 1 & 48 & 1,360 & 69 & 51 \\
Mars & 2.5 & 1 & $30_{-20}^{+60}$ & $280 \pm 70$ & $110_{-70}^{+220}$ & $250 \pm 60$ \\
CI Chondrite & 3.3 & 1 & 109 & 114 & 30 & 605 \\
\hline
\end{tabular}

* Adapted from Donahue and Pollack (1987) and Anders and Grevesse (1989).

Wetherill (1981) took the position that solar wind implantation was the basic source for all of the Venusian noble gases and that selective escape of neon led to the present abundance pattern. We suggest instead that solar wind irradiation was much less intense or efficient and that, in fact, only the neon that we now find on Venus was contributed this way. The other noble gases that would have been implanted by the solar wind form a negligible fraction of the abundance we now find in the atmosphere. Some other source is required to provide the large amounts of argon, krypton, and (presumably) xenon. Donahue (1986) attempted to solve this problem by invoking a mechanism for selective loss of noble gases from accreting planetesimals, but admitted that this proposal failed. A new approach, requiring an intense EUV flux from the young Sun to produce massive hydrodynamic escape from a reservoir consisting of a few wt. $\%$ of $\mathrm{H}_{2} \mathrm{O}$, has been more successful (e.g., Hunten et al. 1987, 1988; Zahnle et al. 1990). The question remains whether or not there is any surviving indication of the nature of the carrier(s) that originally brought the volatiles to the planets. If strictly local condensation is invoked, there is a difficulty in obtaining the required amounts of $\mathrm{H}_{2} \mathrm{O}$ for the massive hydrodynamic escape scenarios (Lewis 1970, 1974).

With few exceptions (e.g., Sill and Wilkening 1978), previous approaches to this question have concentrated on various classes of meteorites. This focus is the natural result of a general awareness of meteoritic bombardment and detailed knowledge about abundance patterns and isotopic ratios in noble gases (and other volatile elements) trapped in the meteorites. We know that comets must have impacted the inner planets also, but our knowledge of the composition of these objects is still very rudimentary. Nevertheless, we do have the results from Halley's Comet (e.g., Geiss 1987; Anders and Grevesse 1989), 
and recent laboratory experiments have given a clear picture of the trapping of gases in ice at low temperature. We can therefore state that a plausible candidate for a source of the Ar, $\mathrm{Kr}$, and $\mathrm{Xe}$, but not $\mathrm{Ne}$, is icy planetesimals - comets - that formed in the outskirts of the solar nebula at low temperatures and bombarded the inner planets during their early history. Such an explanation will have to satisfy both the overall abundances and the ${ }^{84} \mathrm{Kr} /{ }^{36} \mathrm{Ar}$ and ${ }^{132} \mathrm{Xe} /{ }^{36} \mathrm{Ar}$ ratios.

\section{Experimental Results}

Recently, we studied the trapping in amorphous ice of mixtures of $\mathrm{CO}, \mathrm{CH}_{4}, \mathrm{~N}_{2}$, and Ar for comparison with the gas content of Comet P/Halley (Bar-Nun et al. 1988). (This paper and the references it contains may be consulted for details about experimental procedures.) We concluded that in order to trap the observed $3.5 \%$ of $\mathrm{CO}$ and $1 \%$ of $\mathrm{CH}_{4}$ in Halley's ices, its cometesimals had to be formed in a region of the solar nebula where the temperature was $48 \pm 2 \mathrm{~K}$ and the $\mathrm{CO} / \mathrm{CH}_{4}$ ratio was at least 100 (Bar-Nun and Kleinfeld 1989).

As a continuation of this study, trapping of mixtures of $\mathrm{Ar}, \mathrm{Kr}$, and $\mathrm{Xe}$ in amorphous ice was studied experimentally between 30 and $75 \mathrm{~K}$ (Bar-Nun et al., in preparation). (The trapping of $\mathrm{H}_{2}, \mathrm{D}_{2}$, and $\mathrm{Ne}$ in ice has been investigated previously (Laufer et al. 1987). Ne was found to be trapped in the ice only below $20 \mathrm{~K}$.) The release of the noble gases from ice that was codeposited on a cold plate at $50 \mathrm{~K}$ from a $\mathrm{H}_{2} \mathrm{O}: \mathrm{Ar}: \mathrm{Kr}: \mathrm{Xe}=$ 10,000:10,000:8:1 gas mixture, upon its slow warming up, is shown in Figure 1. What is obvious from this plot is the overabundance in the ice of trapped $\mathrm{Kr}$ and Xe over the Ar, as compared with the starting composition of the gas mixture. This overabundance increases as the deposition temperature increases from 30 to $75 \mathrm{~K}$. The enrichment factors in $\mathrm{Kr} / \mathrm{Ar}$ and $\mathrm{Xe} / \mathrm{Ar}$ (their ratios in the ice divided by their ratios in the deposited gas mixture) are shown, for various temperatures, in Table 2.

These enrichment factors are strongly temperature-dependent, since above $30 \mathrm{~K}$, there is a competition among the gases for the available trapping sites in the amorphous ice, and the more polarizable $\mathrm{Kr}$ and Xe have an advantage over the Ar. It should be noted that a ratio of $\mathrm{Ar}: \mathrm{Kr}: \mathrm{Xe}=10,000: 8: 1$ is the solar ratio within a factor of 2, which also prevailed in the solar nebula (Table 3). A hundredfold dilution with $\mathrm{CO}$, as expected in the outer reaches of the nebula, did not affect these enrichment factors.

\section{Discussion}

After establishing the noble gas ratios on the terrestrial planets (Table 1) and the ratios of these gases in the amorphous ice as a function of its formation temperature (Table 2), we can compare the two and find whether comets could have delivered $\mathrm{Ar}, \mathrm{Kr}$, and $\mathrm{Xe}$ to these planets. Below $30 \mathrm{~K}$, there is no enrichment-the gases are trapped in the ice at the same ratios they exhibit in the gas phase. Hence, for Venus, solar values of ${ }^{84} \mathrm{Kr} / 36 \mathrm{Ar}$ and ${ }^{132} \mathrm{Xe} / 36 \mathrm{Ar}$ can be obtained from bombardment by a comet formed at $\leq 30 \mathrm{~K}$. The enrichments at $38 \mathrm{~K}$ are already too high. The enrichment of ${ }^{84} \mathrm{Kr} / 36 \mathrm{Ar}$ on Mars and Earth can be provided by a comet formed at $52 \mathrm{~K}$, but the ${ }^{132} \mathrm{Xe} / 36 \mathrm{Ar}$ enrich- 


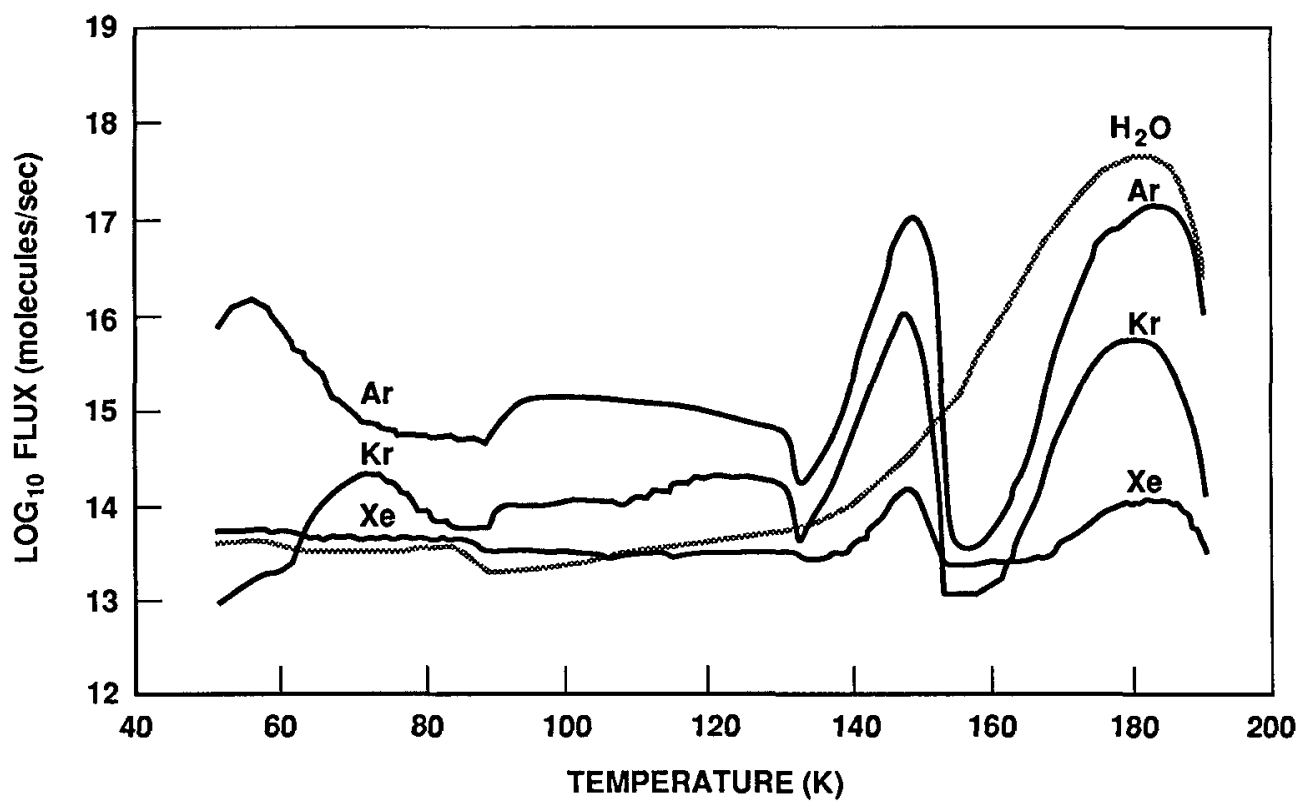

Figure 1. A plot of the fluxes of gases and water vapor vs. temperature. The gas-rich amorphous ice was conducted at $50 \mathrm{~K}$ from a $\mathrm{H}_{2} \mathrm{O}: \mathrm{Ar}: \mathrm{Kr}: \mathrm{Xe}=10,000: 10,000: 8: 1 \mathrm{mix}-$ ture. Note the relative enrichment of $\mathrm{Kr}$ and Xe over Ar.

ment cannot be obtained at this temperature. A temperature of $57 \mathrm{~K}$ would fit the ${ }^{132} \mathrm{Xe} / 36 \mathrm{Ar}$, but would increase the ${ }^{84} \mathrm{Kr} / 36 \mathrm{Ar}$ by an unacceptable amount.

It seems to us that we can therefore explain at least part of the noble gas inventories of the atmospheres of the inner planets in terms of impacts with two types of icy planetesimals. Venus requires an impactor composed of ices that have formed at temperatures between 20 and $30 \mathrm{~K}$. At lower temperatures, there would be too much neon. At higher temperatures, the krypton and xenon would be enriched relative to their values in the solar nebula. On the other hand, it seems that only the argon and krypton on Mars and Earth could have come from a cometary source; the xenon requires a different origin. This might be the signal of the meteoritic contribution. Note in Table 1 that $\mathrm{C} 1$ chondrites are more enriched in $\mathrm{Xe} / \mathrm{Ar}(=605)$ than in $\mathrm{Kr} / \mathrm{Ar}(=30)$. Hence, if such meteorites supplied the observed amounts of Xe on both planets, their contribution to the other noble gases would 
Table 2. Enrichment Factors Obtained Experimentally by Codepositing a Mixture of $\mathrm{H}_{2} \mathrm{O}: \mathrm{Ar}: \mathrm{Kr}: \mathrm{Xe}=10,000: 10,000: 8: 1$

\begin{tabular}{|c|c|c|c|}
\hline Ice Deposition & \multicolumn{2}{|c|}{ Enrichment Factors* } & Total Amount of \\
\hline 30 & 1 & 1 & -3 \\
\hline 38 & 16 & 7 & 1.25 \\
\hline 50 & 56 & 19 & 0.32 \\
\hline 54 & 92 & 25 & 0.216 \\
\hline 60 & 365 & 83 & 0.091 \\
\hline 70 & 809 & 779 & 0.017 \\
\hline 75 & 3714 & 3680 & 0.0031 \\
\hline
\end{tabular}

* Enrichment factors are defined as the ratio $\mathrm{Kr} / \mathrm{Ar}$ or $\mathrm{Xe} / \mathrm{Ar}$ trapped in the ice, divided by the ratio $\mathrm{Kr} / \mathrm{Ar}$ or $\mathrm{Xe} / \mathrm{Ar}$ in the gas mixture, which was deposited on the cold plate.

be well below the cometary one. Alternatively, we may be seeing here the effects of early hydrodynamic escape (Zahnle et al. 1990).

As to the sizes of these impacting comets, if Earth's entire atmospheric ${ }^{36} \mathrm{Ar}+{ }^{38} \mathrm{Ar}$ and $\mathrm{Kr}$ were brought by a single comet that formed at $\sim 52 \mathrm{~K}$ in a nebula of the composition shown in Table 3 and that had a density of $0.5 \mathrm{~g} \cdot \mathrm{cm}^{-3}$, the comet's radius would have to be only $42 \mathrm{~km}$. This is only twice the current estimate for Comet SchwassmannWachmann 1, and less than half the putative size of Chiron $(\mathrm{R} \sim 100 \mathrm{~km})$. At $30 \mathrm{~K}$, the comet would contain about 30 times more gas than one formed at $50 \mathrm{~K}$ (Table 2). Hence, for Venus, a $\mathrm{R}=63 \mathrm{~km}$ comet is sufficient to supply the 100 times more $36 \mathrm{Ar}$ and the accompanying $\mathrm{Kr}$ and Xe. To supply Mars with its 100 times less ${ }^{36} \mathrm{Ar}$ and $\mathrm{Kr}, \mathrm{a}$ $\mathrm{R}=8 \mathrm{~km}$ comet is sufficient.

We return now to the source of the Venusian neon. As shown above, Ne is trapped in the ice only below $20 \mathrm{~K}$ and could not have been delivered by comets. Support for solar irradiation of preplanetary grains can be mustered by a comparison with the neon implanted in lunar soils. Here, an average value of $20 \mathrm{Ne} / 22 \mathrm{Ne}=12.3$ has been found, consisting of two components: solar-wind neon, with ${ }^{20} \mathrm{Ne} / 22 \mathrm{Ne}=13.7 \pm 0.3$, and solar-flare neon, with a ratio of $11.3 \pm 0.3$ (see Anders and Grevesse (1989) for a discussion). The early solar wind was more intense than it is today, but the greater activity of 
Table 3. Abundances of Gases in the Solar Nebula, Relative to $\mathrm{H}_{2}{ }^{*}$

\section{All $\mathrm{C}$ as $\mathrm{CH}_{4} \quad$ C-Independent Values $\quad$ All $\mathrm{C}$ as $\mathrm{CO}$}

$\begin{array}{lccc}\mathrm{H}_{2} \mathrm{O}^{* *} & 1.7 \times 10^{-3} & & 1.0 \times 10^{-3} \\ \mathrm{CH}_{4} & 7.2 \times 10^{-4} & & 0 \\ \mathrm{CO} & 0 & 1.1 \times 10^{-4} & 7.2 \times 10^{-4} \\ \mathrm{~N}_{2} & & 2.5 \times 10^{-4} & \\ \mathrm{Ne} & 7.2 \times 10-6 & \\ \mathrm{Ar} & & 3.3 \times 10^{-9} & \\ \mathrm{Kr} & 3.2 \times 10^{-10} & \\ \mathrm{Xe} & & & \end{array}$

* Calculated from the atomic abundances of Anders and Grevesse (1989), assuming that the molecules listed are the major ones in the nebula.

** If other oxygen-bearing molecules, such as $\mathrm{MgO}$, are taken into account, the $\mathrm{H}_{2} \mathrm{O}$ abundance should be lower by about $20 \%$.

the young Sun suggests that there was a higher proportion of solar-flare neon at that time, an expectation that is supported by the data from lunar samples (Wieler et al. 1983). Given that the higher energy solar-flare neon is better retained by the irradiated grains, there seems to be little difficulty in reaching the value of ${ }^{20} \mathrm{Ne} / 22 \mathrm{Ne}=11.9 \pm 0.7$ reported for Venus (Istomin et al. 1982).

Finally, we can make a crude estimate of the efficiency of solar-wind implantation of the preplanetary grains by again referring to the bulk lunar samples (Wieler et al. 1983). We find that the total $20 \mathrm{Ne}$ in 11 samples averages (with a wide range) to a value of $150 \times 10^{-5} \mathrm{~cm}^{3} \cdot \mathrm{g}^{-1}$ or $150 \times 10^{-14} \mathrm{~cm}^{3} \cdot \mathrm{g}^{-1} \cdot \mathrm{yr}^{-1}$. Adopting Wetherill's (1981) time scale of 105 years for the solar-wind irradiation of preplanetary material in the orbit of Venus, this lunar analogue model predicts a neon abundance of $3 \times 10^{-7} \mathrm{~cm}^{3} \cdot \mathrm{g}^{-1}$ in the Venusian atmosphere, assuming all the neon that was implanted in the grains is now in the atmosphere. The observed value is $5 \times 10^{-7} \mathrm{~cm}^{3} \cdot \mathrm{g}^{-1}$, which is in closer agreement than one could reasonably expect for such an approximate calculation. 


\section{Conclusions}

The idea that comets could have brought volatiles to the forming inner planets is not new (e.g., Oro 1961, Chyba 1987), but it has been difficult to distinguish cometary from meteoritic contributions in the volatile inventories currently available on the planets. We suggest that the amounts and relative abundances of noble gases in the atmosphere of Venus may offer the first clear indication of the presence of a cometary component in a planetary atmosphere. This component could have been provided by the impact of an icy planetesimal with a radius of $60 \mathrm{~km}$ formed at temperatures between 20 and $30 \mathrm{~K}$.

We propose a single impact rather than a family of small ones, since the model must also explain why Earth and Mars do not show the same pattern. All of the inner planets were presumably subjected to roughly the same flux of impacting meteorites and comets. The idea here is that visits to the inner solar system by such low-temperature comets were relatively rare. Thus either Venus was the only inner planet to receive such a hit, or the records of such hits on Earth and (especially) on Mars were removed by subsequent atmosphere-eroding bombardment (Cameron 1983, Melosh and Vickery 1989). The issue, then, becomes one of timing, rather than one of the uniqueness of the impactor. While this may seem rather ad hoc, several of the discoveries made by Voyager's exploration of the outer solar system and the current consensus for the impact-formation of our planet's moon have emphasized the importance of singular events in determining the characteristics of both planets and satellites.

A comet formed at higher temperatures ( $~ 50$ to $55 \mathrm{~K}$ ) could account for argonkrypton or krypton-xenon patterns found on Earth and Mars, but this interpretation is not justified, since a single formation temperature no longer fits the data. On the other hand, impacts of comets must have occurred on Mars, and our demonstration that a body as small as $\mathrm{R}=8 \mathrm{~km}$ could contribute the present amounts of $\mathrm{Kr}$ and $\mathrm{Ar}$ suggests that a cometary contribution to the Martian atmosphere may yet be distinguished. The ${ }^{20} \mathrm{Ne} / 22 \mathrm{Ne}$ values on Earth and (probably) Mars also require a special explanation that is perhaps provided by hydrodynamic escape (e.g., Zahnle et al. 1990). On Venus, it appears that irradiation of preplanetary material by an intense early solar wind can satisfactorily account for both the abundance and the isotopic ratio of the atmospheric neon.

This model can easily be tested by investigating noble gases associated with icy objects. The absence of detectable neon in the atmosphere of Titan is certainly consistent with this discussion, but hardly adds to it (Owen 1982, 1985). The proposed missions to Comet Kopff (Comet Rendezvous Asteroid Flyby [CRAF]) and Titan (Cassini-Huyghens), planned for arrivals early in the next century, should provide the definitive data. Meanwhile, the Galileo probe will tell us the value of the neon isotope ratio in Jupiter's atmosphere, which should represent the starting value in the primordial solar nebula. 


\section{References}

Anders, E., and Grevesse, N. (1989). 'Abundances of the elements: Meteoritic and solar.' Geochim. et Cosmochim. Acta 53, 197-214.

Anders, E., and Owen, T. (1977). 'Origin and abundances of volatiles on the Earth and Mars.' Science 198, 453-465.

Bar-Nun, A., and Kleinfeld, I. (1989). 'On the temperature and composition in the region of comet formation.' Icarus 80, 243-253.

Bar-Nun, A., Kleinfeld, I., and Kochavi, E. (1988). 'Trapping of gas mixtures by amorphous water ice.' Phys. Rev. B. 38, 7749-7754.

Cameron, A.G.W. (1983). 'Origin of the atmospheres of the terrestrial planets.' Icarus $56,195-201$.

Chyba, C. (1987). 'The cometary contribution to the oceans of primitive Earth.' Nature $330,632-635$.

Donahue, T.M. (1986). 'Fractionation of noble gases by thermal escape from accreting planetesimals.' Icarus $66,195-212$.

Donahue, T.M., and Pollack, J.B. (1983). 'Origin and evolution of the atmosphere of Venus.' In Venus, D.M. Hunten, L. Colin, T.M. Donahue, and V.I. Moroz (eds.), University of Arizona Press, Tucson, pp. 1003-1036.

Geiss, J. (1987). 'Composition measurements and the history of cometary matter.' Astron. Astrophys. 187, 859-866.

Hunten, D.M., Pepin, R.O., and Walker, J.C.G. (1987). 'Mass fractionation in hydrodynamic escape.' Icarus 69, 532-549.

Hunten, D.M., Pepin, R.O., and Owen, T. (1988). 'Planetary atmospheres.' In Meteorites and the Early Solar System, J.F. Kerridge and M.S. Matthews (eds.), University of Arizona Press, Tucson, pp. 565-594.

Istomin, V.G., Grechnev, K.V., and Kochnev, J.V.A. (1982). 'Preliminary results of mass-spectrometric measurements on board the Venera 13 and 14 probes.' Pism Astron. Zh. 8, 391-398.

Laufer, D., Kochavi, E., and Bar-Nun, A. (1987). 'Structure and dynamics of amorphous water ice.' Phys. Rev B. 36, 9219-9227.

Lewis, J.S. (1970). 'Venus: Atmospheric and lithospheric composition.' Earth Planet. Sci. Lett. 10, 73-80.

Lewis, J.S. (1974). 'The temperature gradient in the solar nebula.' Science 186, 440443.

Melosh, J., and Vickery, A. (1989). 'Impact erosion of the primordial Martian atmosphere.' Nature 338, 487-489.

Oro, J. (1961). 'Comets and the formation of biochemical compounds on the primitive Earth.' Nature 190, 389-390.

Owen, T. (1982). 'The composition and origin of Titan's atmosphere.' Planet. Space Sci. $30,833-838$.

Owen, T. (1985). 'The atmospheres of icy bodies.' In Ices in the Solar System, J. Klinger, D. Benest, A. Dollfus, and R. Smoluchowski (eds.), D. Reidel Publishing Co., Dordrecht, pp. 731-740.

Sill, G., and Wilkening, L. (1978). 'Ice clathrate as a possible source of the atmospheres of the terrestrial planets.' Icarus 33, 13-22.

Wetherill, G.W. (1981). 'Solar wind origin of ${ }^{36} \mathrm{Ar}$ on Venus.' Icarus 46, 70-80. 
Wieler, R., Etique, Ph., Signer, P., and Poupean, G. (1983). 'Decrease of the solar flare/solar wind flux ratio on the past several aeons deduced from solar neon and tracks in lunar soil plagioclases.' J. Geophys. Res. 88 Supp., A713-A724.

Wiens, R.C., Becker, R.H., and Pepin, R.O. (1986). 'The case for Martian origin of the shergottites II. Trapped and indigenous gas components in EETA 79001 glass.' Earth Planet. Sci. Lett. 77, 149-158.

Zahnle, K.J., Kasting, J.F., and Pollack, J.B. (1990). 'Mass fractionation of noble gases in diffusion-limited hydrodynamic hydrogen escape.' Icarus 84, 502-527. 